Polymer Journal Vol. 1, No. 6, pp 616-631 (1970)

\title{
Hydrodynamic Properties of Rigid Complex Molecules
}

\author{
Keishiro TsudA \\ Research Institute for Polymers and Textiles, Sawatari, \\ Kanagawa-ku, Yokohama, Japan.
}

(Received December 25, 1969)

\begin{abstract}
Three hydrodynamic properties - the translational frictional coefficient, the rotational frictional coefficient, and the intrinsic viscosity-for a model molecule composed of different frictional elements in an arbitrary conformation are calculated by a tensor method. The method adopts the Oseen tensor rigorously and differs from Kirkwood's in the respect that the present method is a unified one for a rigid molecule, but gives the same result for the translational frictional coefficient. The method is extended to Bloomfield's "shell model". In the case of a sphere, the present method gives the Stokes-Kirchhoff law for the rotational frictional coefficient and the Einstein relation for the intrinsic viscosity. The present theory agrees well with experiments. For bovine serum albumin, the hydrodynamic properties are more sensitive to the difference of molecular structures in the order, the translational frictional coefficient, the intrinsic viscosity, and the rotational frictional coefficient. For $\gamma$ globulin, the Edelman-Gally model is shown to be consistent with the hydrodynamic properties at various stages of degradation.
\end{abstract}

KEY WORDS Tensor Method/Oseen Tensor/Translational Frictional Coefficient/Rotational Frictional Coefficient/Intrinsic Viscosity/ Shell Model / Bovine Serum Albumin/Gamma Globulin /

The purpose of this study is to calculate three hydrodynamic properties-the translational frictional coefficient, the rotational frictional coefficient, and the intrinsic viscosity-of rigid complex molecules by a unified method. The method consists of derivation of fundamental equations by a unified tensor method and their application to the "shell model". ."

The works forming the basis of the present study are the Kirkwood equation ${ }^{2}$ and the Bloomfield "shell model", for the translational frictional coefficient. The Kirkwood equation for the translational frictional coefficient ${ }^{2}$ is that for a molecule of identical frictional elements in an arbitrary conformation and obtained with a tensor method by use of the rigorous form of the Oseen hydrodynamic interaction tensor ${ }^{3}$. Furthermore, its remarkable characteristic revealed by Bloomfield, is that it gives the Stokes law for a sphere on the basis of the shell model. ${ }^{1}$ The shell model for the translational frictional coefficient ${ }^{1}$ utilizes the Kirkwood equation as a fundamental equation and deals with complex molecules of arbitrary shape. On the other hand, the shell model, ${ }^{4}$ based on the Hearst equation, ${ }^{5}$ for the rotational frictional coefficient is that for molecules of cylindrical symmetry and not of arbitrary shape. For the intrinsic viscosity, the shell model has not been reported except in our previous works. ${ }^{6}$ Such a situation in the rotational frictional coefficient and the intrinsic viscosity may be partly ascribed to the fact that the fundamental equations corresponding to the Kirkwood equation, suitable to the shell model, were not proposed.

We first derive the fundamental equations for the three hydrodynamic properties corresponding to the Kirkwood equation by a unified method. The requirements of the method are (1) to deal with a rigid molecule composed of frictional elements in an arbitrary conformation, (2) to take rigorously the Oseen tensor into account and (3) to use a unified tensor method. Besides, we use two kinds of approximation; first we omit the component of the force normal to the direction of motion of the molecule; secondly in a final procedure of averaging, we replace the quantities directly related to the basic vectors 


\section{Hydrodynamic Properties of Rigid Complex Molecules}

by the averaged values of the quantities. As shown in the following part, the first approximation reduces to the second one. An approximation similar to the second one has been used by Hearst. ${ }^{5}$ With these requirements and approximations we have the Kirkwood equation for the translational frictional coefficient and at the same time satisfy the criteria that the fundamental equations give for a sphere on the use of the shell model, the Stokes-Kirchhoff law for the rotational frictional coefficient and the Einstein relation for the intrinsic viscosity. ${ }^{7}$ Here the unified tensor method means that we obtain all three hydrodynamic properties by a tensor method, while Kirkwood and others have calculated the frictional coefficients by a tensor method,,$^{2,8,9}$ but the intrinsic viscosity by solving the generalized diffusion equation of the distribution function. ${ }^{2,10}$ Here we treat the case in which we are allowed to ignore the effect of Brownian motion, but they consider the effect rigorously. The present unified tensor method is obtained by treatment of a rigid model without taking Brownian motion into account.

Secondly, the fundamental equations derived above are applied to the shell model for a molecule of different spherical subunits. Then we have the equations for the shell model of the rotational frictional coefficient and of the intrinsic viscosity respectively, which correspond to Bloomfield's for the translational frictional coefficient. The shell model ${ }^{1,11}$ enables us to investigate the hydrodynamic properties on the basis the model closely approximates the actual shape of a complex molecule; the approximation can be made as precise as we need by selecting the size and the conformation of the subunits. Accordingly, detailed comparison of the hydrodynamic model with models obtained from other procedures, such as electron microscopy and X-ray diffraction, becomes possible. This problem has been for many years one of great concern to biochemists.

The last part is the comparison of the theory with experiments for bovine serum albumin and $\gamma$ globulin. Bovine serum albumin is used to study the relation between the structural difference of the molecule and the three hydrodynamic properties. $\gamma$ globulin is adopted to compare hydrodynamic models with models obtained from other procedures such as electron microscopy and to test the Edelman-Gally model $^{34}$ at various stages of degradation.

\section{THEORY}

\section{Unified Tensor Method*}

Some Basic Equations. The fundamental equations for the three hydrodynamic properties of a rigid molecule are calculated by a unified tensor method. The equations correspond to the Kirkwood equation ${ }^{2,8,9}$ for the translational frictional coefficient of a molecule composed of identical frictional elements in an arbitrary conformation and are obtained by rigorous use of the Oseen tensor. We start with the following basic equations.

Let us consider as a model of a macromolecule, a rigid assembly of $n$ identical frictional elements in an arbitrary conformation in a fluid flow. The force exerted on the fluid by the $l$ th element located at the point $\boldsymbol{R}_{l}$ from the molecular centre of mass, is given by

$$
\boldsymbol{F}_{l}=-\zeta\left(\boldsymbol{v}_{l}-\boldsymbol{u}_{l}\right)
$$

where $\zeta$ is the friction constant of a single element, $\boldsymbol{u}_{l}$ is the velocity of the $l$ th element and $\boldsymbol{v}_{l}$ is the solvent velocity at $\boldsymbol{R}_{l}$. The velocity $\boldsymbol{v}_{l}$ is calculated from the Oseen formula, with consideration of the hydrodynamic perturbations due to other elements: ${ }^{3}$

$$
\begin{aligned}
\boldsymbol{v}_{l} & =\boldsymbol{v}_{l}{ }^{0}-\zeta \sum_{\substack{s=1 \\
s \neq l}}^{n} \boldsymbol{T}_{l s} \cdot \boldsymbol{F}_{s} \\
\boldsymbol{T}_{l l} & =0 \\
\boldsymbol{T}_{l s} & =\frac{1}{8 \pi \eta_{0} \boldsymbol{R}_{l s}}\left(1+\frac{\boldsymbol{R}_{l s} \boldsymbol{R}_{l s}}{\boldsymbol{R}_{l s}^{2}}\right), \quad l \neq s \\
\boldsymbol{R}_{l s} & =\boldsymbol{R}_{s}-\boldsymbol{R}_{l}, \quad \boldsymbol{R}_{l s}=\left|\boldsymbol{R}_{l s}\right|
\end{aligned}
$$

where $\boldsymbol{v}_{l}{ }^{0}$ is the unperturbed velocity at $\boldsymbol{R}_{l}$ in the absence of the assembly, $\boldsymbol{T}_{l s}$ is the Oseen tensor in the Stokes approximation, and $\eta_{0}$ is the viscosity of the solvent.

The substitution of eq 2 and 3 in eq 1 gives

$$
\boldsymbol{F}_{l}=\zeta\left(\boldsymbol{u}_{l}-\boldsymbol{v}_{l}{ }^{0}\right)-\zeta \sum_{s=1}^{n} \boldsymbol{T}_{l s} \cdot \boldsymbol{F}_{s}
$$

* Reported, in part: K. Tsuda, Pept. Progr. Polym. Phys. Japan, 12, 55 (1969). 


\section{K. TSUDA}

This equation is rewritten as

$$
\boldsymbol{F}=\zeta\left(\boldsymbol{u}-\boldsymbol{v}^{0}\right)-\zeta \boldsymbol{T} \cdot \boldsymbol{F}
$$

with

$$
\begin{aligned}
& \boldsymbol{F}=\sum_{l=1}^{n} \boldsymbol{F}_{l}, \quad \boldsymbol{T}=\sum_{\substack { l=1 \\
\begin{subarray}{c}{s=1 \\
s \neq l{ l = 1 \\
\begin{subarray} { c } { s = 1 \\
s \neq l } }\end{subarray}}^{n} \boldsymbol{T}_{l s}, \\
& \boldsymbol{u}=\sum_{l=1}^{n} \boldsymbol{u}_{l}, \quad \boldsymbol{v}^{0}=\sum_{l=1}^{n} \boldsymbol{v}_{l}^{0}
\end{aligned}
$$

In the following calculation, we employ the approximations that we omit the tensor components normal to the direction of the motion of the molecule and that, in a final averaging procedure, we replace the quantities directly related to the basic vectors by the averaged values of the quantities. The first approximation is due to the symmetry of the allowable orientations of the molecule in averaging and is implied in the second; hence, the second is essential. An approximation of the average is not necessary for the translational frictional coefficient but inevitable for the rotational frictional coefficient and the intrinsic viscosity to avoid difficulty in the mathematics. The approximation adopted above is shown to be sufficient for the deduction of the Kirkwood equation for the translational frictional coefficient; the Kirchhoff law for the rotational frictional coefficient, and the Einstein relation for the intrinsic viscosity for a sphere by use of the shell model.

Translational Frictional Coefficient. We consider a resistance force exerted on a fluid at rest by a motion of a rigid molecule with a constant velocity in a direction $\lambda$. The force generally consists of components along and normal to the $\lambda$ direction and the components become equal to the friction tensor's when the velocity is a unit velocity $\boldsymbol{e}_{\lambda}$. In this case, we have $e_{\lambda}=\boldsymbol{u}_{l}-\boldsymbol{v}_{l}{ }^{0}$ in eq 5 , because the velocity of every frictional element of the molecule is equal in a rigid model. If we define a covariant vector as $\boldsymbol{a}_{\lambda}=\sum_{l=1}^{n} \boldsymbol{e}_{\lambda}$, we have from eq 6

$$
\boldsymbol{F}=\zeta \boldsymbol{a}_{\lambda}-\zeta \boldsymbol{T} \cdot \boldsymbol{F}
$$

This equation is solved as

or

$$
\boldsymbol{F}=\zeta(1+\zeta \boldsymbol{T})^{-1} \boldsymbol{a}_{\lambda}
$$

$$
\boldsymbol{F}=\mathscr{F} \boldsymbol{a}_{\lambda}
$$

with the abbreviation of the operator

$$
\mathscr{F}=\zeta(1+\zeta T)^{-1}
$$

Therefore, eq 8 is rewritten as

$$
\mathscr{F} \boldsymbol{a}_{\lambda}=\zeta \boldsymbol{a}_{\lambda}-\zeta \boldsymbol{T} \mathscr{F} \boldsymbol{a}_{\lambda}
$$

To obtain a mean frictional coefficient, we need to average, in a final procedure, the friction tensor over all orientations of the molecule. On account of the symmetry of the orientations, the components of friction tensor normal to the $\lambda$ direction should vanish and only the component in the $\lambda$ direction is considered:

$\zeta_{\lambda \lambda}=\boldsymbol{a}_{\lambda} \cdot \boldsymbol{F}=\boldsymbol{a}_{\lambda} \mathscr{F} \boldsymbol{a}_{\lambda}=\zeta \boldsymbol{a}_{\lambda} \cdot \boldsymbol{a}_{\lambda}-\zeta \boldsymbol{a}_{\lambda} \boldsymbol{T} \mathscr{F} \boldsymbol{a}_{\lambda}$

Further, in the above meaning of average, a friction tensor due to the motion of the molecule in any direction, if the space used is isotropic, will give the same result; $\zeta_{\lambda \lambda}$ is equal regardless of $\lambda$. Therefore, we may choose an arbitrary direction for $\lambda$.

In this first approximation, we ignore the tensor components normal to the direction of motion from the beginning of the calculation: that is, we consider the space constructed only by the $\lambda$ component. ${ }^{*}$ Then we have the metric tensor, its contravariant tensor, and the contravariant vector reciprocal to $\boldsymbol{a}_{\lambda}$ as

$$
\boldsymbol{g}_{\lambda \lambda}=\boldsymbol{a}_{\lambda} \cdot \boldsymbol{a}_{\lambda}, \quad g^{\lambda \lambda}=\frac{1}{\boldsymbol{a}_{\lambda} \cdot \boldsymbol{a}_{\lambda}}, \quad \boldsymbol{a}^{\lambda}=g^{\lambda \lambda} \boldsymbol{a}_{\lambda}
$$

Here the fundamental metric of the space considered is a product of the covariant vectors, $\boldsymbol{a}_{\lambda}$ 's, and the covariant vectors are a set of vectors referred to as basic vectors. ${ }^{2}$ If the basic vector is denoted by $\boldsymbol{b}_{\lambda}{ }^{(l)}$, we have in the case of this section, that $\boldsymbol{b}_{\lambda}{ }^{(l)}=\boldsymbol{e}_{\lambda}$ or $\boldsymbol{a}_{\lambda}=\sum_{l=1}^{n} \boldsymbol{b}_{\lambda}{ }^{(l)}=$ $\sum_{l=1}^{n} \boldsymbol{e}_{\lambda}$. A similar definition of the basic vector is used in the following sections.

$\mathrm{Eq} 13$ is rewritten as

$$
\zeta_{\lambda \lambda}=\zeta \boldsymbol{a}_{\lambda} \cdot \boldsymbol{a}_{\lambda}-\zeta \boldsymbol{a}_{\lambda} \boldsymbol{T a}^{\lambda} \boldsymbol{a}_{\lambda} \mathscr{F} \boldsymbol{a}_{\lambda}=\zeta g_{\lambda \lambda}-\zeta T_{\lambda}^{\lambda} \zeta_{\lambda \lambda}
$$

* A similar approximation has been used by Burgers $^{3}$ and Ishihara (A. Ishihara, Fortsch. Hochpolym. Forsch., 5, 531 (1968).) for the translational frictional coefficient of a sphere. 
Hydrodynamic Properties of Rigid Complex Molecules

where

$$
a_{\lambda} T a^{\lambda}=T_{\lambda}^{\lambda}
$$

Multiplying eq 15 by $g^{\lambda \lambda}$ and using the relation

$$
\left(\zeta_{\lambda \lambda}\right)\left(\zeta^{-1}\right)^{\lambda \lambda}=1
$$

we obtain

$$
\left(\zeta^{-1}\right)^{\lambda \lambda}=\frac{g^{2 \lambda}}{\zeta}+T^{\lambda \lambda}
$$

or

$$
\zeta_{\lambda \lambda}=\frac{1}{\frac{g^{\lambda \lambda}}{\zeta}+T^{\lambda \lambda}}
$$

Eq 18 corresponds to the mobility tensor of Kirkwood. ${ }^{8}$ Choosing as $e_{\lambda}$, for example, the unit vector $\boldsymbol{e}_{x}$ in the direction of $x$ axis, we average the friction tensor $\zeta_{x x}$ over all orientations and internal coordinates of the molecule to obtain a mean translational frictional coefficient. Here we replace the quantities such as the fundamental metric, directly related to the basic vectors by the averaged values of the quantities as Hearst ${ }^{5}$ did for the rotatory diffusion constants of stiff-chain macromolecules.* This second approximation for average yields eq 18 and 19 from the general solution of Kirkwood $^{2,8,9}$ which considers the components normal to the direction of motion as well. Thus, the first approximation is seen to be included in the second. On the basis of the relation between a mean frictional coefficient and a mean diffusion constant:

$$
f_{t}=\frac{k T}{\bar{D}}
$$

where $k$ is the Boltsmann constant and $T$ the absolute temperature, we take a harmonic mean

* The procedure of averaging of $\left(\zeta^{-1}\right)^{\alpha \beta}$ used by Hearst ${ }^{5}$ is in effect as follows. We first obtain: $\overline{\boldsymbol{g}_{\alpha \beta}}=\overline{\boldsymbol{a}_{\alpha} \cdot \boldsymbol{a}_{\beta}}=\sum_{l=1}^{n}\left\langle\boldsymbol{b}_{\alpha}(l) \cdot \boldsymbol{b}_{\beta}(l)\right\rangle$. Then we have: $\overline{\boldsymbol{g}}=$ $\left|\overline{g_{\alpha \beta}}\right|, \quad \overline{g^{\alpha \beta}}=\frac{|\bar{g}|_{\alpha \beta}}{\bar{g}}$, and $\overline{T_{l s}^{\alpha \beta}}=\frac{1}{8 \pi \eta_{0} R_{l s}} \sum_{\sigma} \sum_{\nu} \overline{g^{\alpha \sigma}}$. $\overline{\boldsymbol{g}^{\beta \nu}}\left[\left\langle\boldsymbol{b}_{\sigma}^{(l)} \cdot \boldsymbol{b}_{\nu}^{(s)}\right\rangle+\frac{\left\langle\left(\boldsymbol{R}_{l s} \cdot \boldsymbol{b}_{\sigma}^{(l)}\right)\left(\boldsymbol{R}_{l s} \cdot \boldsymbol{b}_{\nu}(s)\right)\right\rangle}{\boldsymbol{R}_{l s}^{2}}\right] . \quad$ With these results, we have $\frac{\overline{g^{\alpha \beta}}}{\zeta}+\overline{T^{\alpha \beta}}=\left(\overline{\frac{g^{\alpha \beta}}{\zeta}+T^{\alpha \beta}}\right)=$ $\left(\overline{\left.\zeta^{-1}\right)^{\alpha \beta}}\right.$. This procedure is referred to as the second approximation and is used throughout in the present report. as an average as Kirkwood did, ${ }^{* *}$

$$
\begin{aligned}
f_{t} & =\frac{1}{\left(\zeta^{-1}\right)^{x x}}=\frac{1}{\left(\frac{g^{x x}}{\zeta}+\overline{T^{x x}}\right)} \\
& =\left(\frac{1}{n \zeta}+\frac{1}{n^{2}} \sum_{\substack { l \\
\begin{subarray}{c}{s=l \\
s=l{ l \\
\begin{subarray} { c } { s = l \\
s = l } }\end{subarray}}\left\langle\boldsymbol{e}_{x} \boldsymbol{T}_{l s} \boldsymbol{e}_{x}\right\rangle\right)^{-1}
\end{aligned}
$$

Here the average denoted by the angular brackets means a procedure of averaging where we take directly an average of the quantities in the brackets; on the other hand, the average denoted by the bars implies a procedure of averaging where we do not directly take an average of the quantities under the bars.

For the translational frictional coefficient, $g^{x x}$ and $T^{x x}$ have simple forms and so can be averaged even without approximation; here, a usual average gives the same result as the average adopting the second approximation. It is for the rotational frictional coefficient and the intrinsic viscosity that we need the second approximation, because $g^{\alpha \beta}$ and $T^{\alpha \beta}$ for them have complicated forms. However the object of this section is to show that we can derive the same result in spite of our approximations proposed above.

In eq 21 we take first the average over all orientations of the molecule, as Kirkwood did for the mean translational diffusion constant and obtain finally,

$$
f_{t}=\left(\frac{1}{n \zeta}+\frac{1}{6 \pi \eta_{0} n^{2}} \sum_{l=1}^{n} \sum_{s=1}^{n}\left\langle\frac{1}{R_{l s}}\right\rangle\right)^{-1}
$$

where the angular brackets mean an average over internal coordinates only, and the prime on the summation signs denotes the sum over $s$, from 1 to $n$ except $l$. In a rigid molecule, we have that $\left\langle 1 / R_{l s}\right\rangle$ equals $1 / R_{l s}$ and so we do not need the procedure of averaging over internal coordinates. However, with consideration of the general case of treating a flexible molecule as Kirkwood and Riseman did, ${ }^{14}$ we take the average over internal coordinates into account. Eq 22 reduces to the result of Kirkwood ${ }^{2}$ with

** A mean translational diffusion coefficient calculated by Kirkwood $2: \bar{D}=\frac{1}{3} k T\left(\overline{\left(\zeta^{-1}\right)^{x x}+\left(\zeta^{-1}\right)^{y y}+}\right.$ $\left(\overline{\left.\left.\zeta^{-1}\right)^{z z}\right)}\right.$, reduces to $\bar{D}=k T \overline{\left(\zeta^{-1}\right)^{x x}}$, because all the averaged diffusion tensors become equal: $\overrightarrow{D^{x x}}=$ $\overline{D^{y y}}=\overline{D^{z z}}, \overline{\left(\zeta^{-1}\right)^{x x}}=\overline{\left(\zeta^{-1}\right)^{y y}}=\overline{\left(\zeta^{-1}\right)^{z z}}$. 


\section{K. TsudA}

eq 20 .

For a rigid molecule composed of $n$ different frictional elements, where the friction constant of the $l$ th element at $\boldsymbol{R}_{l}$ from the molecular centre of mass is $\zeta_{l}$, the same calculation gives

$f_{t}=\left(\sum_{l=1}^{n} \zeta_{l}\right)\left(1+\frac{1}{6 \pi \eta_{0} \sum_{l=1}^{n} \zeta_{l}} \sum_{l=1}^{n} \sum_{s=1}^{n} \zeta_{l} \zeta_{s}\left\langle\frac{1}{R_{s l}}\right\rangle\right)^{-1}$

This is the same result as Bloomfield's. Here $B$ in the brackets denotes Bloomfield's equations.

Rotational Frictional Coefficient. Let us consider a rotation of a molecule around an arbitrary axis $\mu$ through the molecular centre of mass in a fluid at rest. Its rotational friction tensor is expressed from a torque caused by its motion of a unit angular velocity $\omega_{0}$ around the $\mu$ axis.

In this case, we have that $\boldsymbol{\omega}_{0} \times \boldsymbol{R}_{l}=\boldsymbol{u}_{l}-\boldsymbol{v}_{l}{ }^{0}$ in eq 5. Further, putting $\boldsymbol{a}_{\mu}=\sum_{l=1}^{n} \boldsymbol{b}_{\mu}{ }^{(l)}=\sum_{l=1}^{n} \boldsymbol{\omega}_{0}$ $\times \boldsymbol{R}_{l}$, where $\boldsymbol{b}_{\mu}{ }^{(l)}$ is a basic vector, we have from eq 6 and 10

$$
\mathscr{F} \boldsymbol{a}_{\mu}=\zeta \boldsymbol{a}_{\mu}-\zeta \boldsymbol{T} \mathscr{F} \boldsymbol{a}_{\mu}
$$

Therefore, for the component of the tensor around the $\mu$ axis, we have

$$
\begin{aligned}
& \zeta_{\mu \mu}=\boldsymbol{a}_{\mu} \mathscr{F} \boldsymbol{a}_{\mu}=\zeta \boldsymbol{a}_{\mu} \cdot \boldsymbol{a}_{\mu}-\zeta \boldsymbol{a}_{\mu} \boldsymbol{T} \boldsymbol{a}^{\mu} \boldsymbol{a}_{\mu} \mathscr{F} \boldsymbol{a}_{\mu} \\
& =\zeta a_{\mu} \cdot a_{\mu}-\zeta T_{\mu}{ }^{\mu} \zeta_{\mu \mu}
\end{aligned}
$$

As in the case of translation, we obtain

$$
\left(\zeta^{-1}\right)^{\mu \mu}=\frac{g^{\mu \mu}}{\zeta}+T^{\mu \mu}
$$

If the rotational axis is the $z$ axis, we have

$$
\boldsymbol{a}_{\mu}=\sum_{l=1}^{n} \boldsymbol{\omega}_{0} \times \boldsymbol{R}_{l}=\sum_{l=1}^{n}\left[\left(\boldsymbol{R}_{l} \boldsymbol{e}_{y}\right) \boldsymbol{e}_{x}-\left(\boldsymbol{R}_{l} \boldsymbol{e}_{x}\right) \boldsymbol{e}_{y}\right]
$$

where $\boldsymbol{e}_{x}, \boldsymbol{e}_{y}$ are unit vectors in the direction of the $x$ and $y$ axes. $g^{z z}$ and $T^{z z}$ can not be averaged by a usual method because they have very complicated forms: for example, $g^{z z}=$ $\mathbf{1} /\left(\sum_{l=1}^{n}\left(\boldsymbol{R}_{l} \boldsymbol{e}_{y}\right)^{2}+\sum_{l=1}^{n}\left(\boldsymbol{R}_{l} \boldsymbol{e}_{x}\right)^{2}\right) . \quad$ Therefore, we need an approximation in some way or other. Here, we use the second approximation* and take the average of the tensor over all orientations

\footnotetext{
* See footnote $*$ on $\mathrm{p} 619$.
}

and internal coordinates. Then we have for a mean rotational frictional coefficient,

$$
\begin{aligned}
f_{r} & =\frac{1}{\left(\zeta^{-1}\right)^{z z}}=2\left\{\frac{1}{\zeta \sum_{l=1}^{n}\left\langle\left(\boldsymbol{R}_{l} \boldsymbol{e}_{x}\right)^{2}\right\rangle}\right. \\
& +\frac{1}{\left(\sum_{l=1}^{n}\left\langle\left(\boldsymbol{R}_{l} \boldsymbol{e}_{x}\right)^{2}\right\rangle\right)^{2} \sum_{l=1}^{n} \sum_{s=1}^{n}\left[\left\langle\left(\boldsymbol{R}_{l} \cdot \boldsymbol{e}_{x}\right)\left(\boldsymbol{R}_{s} \boldsymbol{e}_{x}\right)\left(\boldsymbol{e}_{y} \boldsymbol{T}_{l s} \boldsymbol{e}_{y}\right)\right\rangle\right.} \\
& \left.\left.-\left\langle\left(\boldsymbol{R}_{l} \boldsymbol{e}_{x}\right)\left(\boldsymbol{R}_{s} \boldsymbol{e}_{y}\right)\left(\boldsymbol{e}_{x} \boldsymbol{T}_{l s} \boldsymbol{e}_{y}\right)\right\rangle\right]\right\}^{-1}
\end{aligned}
$$

Averaging first over all orientations of the molecule, we have

$$
\begin{aligned}
f_{r} & =\left(\frac{2}{3} \zeta \sum_{l=1}^{n}\left\langle R_{l}{ }^{2}\right\rangle\right) \\
& \times\left\{1+\frac{\zeta}{8 \pi \eta_{0} \sum_{l=1}^{n}\left\langle R_{l}{ }^{2}\right\rangle} \sum_{l=1}^{n} \sum_{s=1}^{n}\left[\left\langle\frac{R_{l} R_{s} \cos \alpha_{l s}}{R_{l s}}\right\rangle\right.\right. \\
& \left.\left.+\left\langle\frac{R_{l}{ }^{2} R_{s}{ }^{2} \sin ^{2} \alpha_{l s}}{2 R_{l s}^{3}}\right\rangle\right]\right\}^{-1}
\end{aligned}
$$

with

$$
\boldsymbol{R}_{l}=\left|\boldsymbol{R}_{l}\right|, \boldsymbol{R}_{s}=\left|\boldsymbol{R}_{s}\right|, \boldsymbol{R}_{l} \boldsymbol{R}_{s} \cos \alpha_{l s}=\left(\boldsymbol{R}_{l} \cdot \boldsymbol{R}_{s}\right)
$$

where the angular brackets mean an average over internal coordinates only.

Eq 29 is derived also from Hearst's result for rotatory diffusion constants, ${ }^{5}$ by taking an average of the components over all orientations of the molecule. Here, the components have already been averaged by him over internal coordinates of the molecules. The coincidence of the results of derivation shows that an average over all orientations and internal coordinates is independent of the order taken. An average over all orientations and over internal coordinates are considered to be equivalent in the sense that both are concerned with the space occupied by the molecule; that is, one relates to the space occupied by the distribution of frictional elements of the molecule and the other to the space occupied by the orientations of the molecule. Further, both are independent of each other. Therefore, in the previous, present, and next sections, we take first the average over all orientations of the molecule.

For a rigid molecule composed of $n$ different frictional elements, we obtain

Polymer J., Vol. 1, No. 6, 1970 
Hydrodynamic Properties of Rigid Complex Molecules

$$
\begin{aligned}
f_{r} & =\left(\frac{2}{3} \sum_{l=1}^{n} \zeta_{l}\left\langle R_{l}{ }^{2}\right\rangle\right) \\
& \times\left\{1+\frac{1}{8 \pi \eta_{0} \sum_{l=1}^{n} \zeta_{l}\left\langle R_{l}{ }^{2}\right\rangle} \sum_{l=1}^{n} \sum_{s=l}^{n} \zeta_{l} \zeta_{s}\right. \\
& \left.\times\left[\left\langle\frac{R_{l} R_{s} \cos \alpha_{l s}}{R_{l s}}\right\rangle+\left\langle\frac{R_{l}{ }^{2} R_{s}{ }^{2} \sin ^{2} \alpha_{l s}}{2 R_{l s}^{3}}\right\rangle\right]\right\}^{-1}
\end{aligned}
$$

The same equation has been derived by a different method in our previous report. ${ }^{13}$

Intrinsic Viscosity. We treat with the intrinsic viscosity at the small shear rate tending to zero. According to Kirkwood and Riseman, ${ }^{14}$ when a solute molecule is introduced into stationary two-dimentional solvent flow with the shear rate $\dot{\varepsilon}$, the intrinsic viscosity is written in the form:

$$
\begin{aligned}
{[\eta] } & =-\frac{N_{0}}{M \eta_{0} \dot{\varepsilon}} \sum_{l=1}^{n}\left\langle\left(\boldsymbol{R}_{l} \cdot \boldsymbol{e}_{y}\right)\left(\boldsymbol{F}_{l} \cdot \boldsymbol{e}_{x}\right)\right\rangle \\
& =-\frac{N_{0}}{M \eta_{0} \dot{\varepsilon}^{2}} \sum_{l=1}^{n}\left\langle\left(\boldsymbol{v}_{l}^{0} \cdot \boldsymbol{F}_{l}\right)\right\rangle=-\frac{N_{0}}{M \eta_{0} \dot{\varepsilon}^{2}}\left\langle\left(\boldsymbol{v}^{0} \cdot \boldsymbol{F}\right)\right\rangle
\end{aligned}
$$

where $N_{0}$ is Avogadro's number and $M$ the molecular weight of the solute. When $\dot{\varepsilon}$ is small or the molecule has a spherical form, it is allowable to put that the angular velocity of the molecule is equal to $\dot{\varepsilon} / 2$ with neglect of the fluctuations due to Brownian motion. Therefore we put

$\boldsymbol{v}_{l}{ }^{0}=\dot{\varepsilon}\left(\boldsymbol{R}_{l} \boldsymbol{e}_{y}\right) \boldsymbol{e}_{x}, \quad \boldsymbol{u}_{l}=\frac{\dot{\varepsilon}}{2}\left[\left(\boldsymbol{R}_{l} \boldsymbol{e}_{y}\right) \boldsymbol{e}_{x}-\left(\boldsymbol{R}_{l} \boldsymbol{e}_{x}\right) \boldsymbol{e}_{y}\right]$

Then the force is written from eq 5 and 33, as

$$
\begin{aligned}
\sum_{l} \boldsymbol{F}_{l}= & -\frac{\zeta \dot{\varepsilon}}{2} \sum_{l}\left[\left(\boldsymbol{R}_{l} \cdot \boldsymbol{e}_{x}\right) \boldsymbol{e}_{y}+\left(\boldsymbol{R}_{l} \boldsymbol{e}_{y}\right) \boldsymbol{e}_{x}\right] \\
& -\zeta \sum_{l} \sum_{s}^{\prime} \boldsymbol{T}_{l s} \boldsymbol{F}_{s}
\end{aligned}
$$

Putting

$$
\begin{aligned}
& \boldsymbol{a}_{\alpha}=\sum_{l} \boldsymbol{b}_{\alpha}^{(l)}=\sum_{l}\left(\boldsymbol{R}_{l} \boldsymbol{e}_{y}\right) \boldsymbol{e}_{x}, \\
& \boldsymbol{a}_{\beta}=\sum_{l} \boldsymbol{b}_{\beta}^{(l)}=\sum_{l}\left[\left(\boldsymbol{R}_{l} \boldsymbol{e}_{x}\right) \boldsymbol{e}_{y}+\left(\boldsymbol{R}_{l} \boldsymbol{e}_{y}\right) \boldsymbol{e}_{x}\right]
\end{aligned}
$$

we have from eq 34

$$
\boldsymbol{F}=-\frac{\zeta \dot{\varepsilon}}{2} \boldsymbol{a}_{\beta}-\zeta \boldsymbol{T} \cdot \boldsymbol{F}
$$

where $\boldsymbol{b}_{\alpha}^{(l)}, \boldsymbol{b}_{\beta}{ }^{(l)}$ are basic vectors and $\boldsymbol{a}_{\alpha}, \boldsymbol{a}_{\beta}$ covariant vectors.

Further, we obtain from eq 36

$$
\boldsymbol{F}=-\frac{\zeta \dot{\varepsilon}}{2}(1+\zeta \boldsymbol{T})^{-1} \boldsymbol{a}_{\beta}=\mathscr{F}^{\prime} \boldsymbol{a}_{\beta}
$$

with the abbreviation of the operator:

$$
\mathscr{F}^{\prime}=-\frac{\zeta \dot{\varepsilon}}{2}(1+\zeta T)^{-1}
$$

Then eq 36 is rewritten as,

$$
\mathscr{F}^{\prime} \boldsymbol{a}_{\beta}=-\frac{\zeta \dot{\varepsilon}}{2} \boldsymbol{a}_{\beta}-\zeta \boldsymbol{T} \mathscr{F}^{\prime} \boldsymbol{a}_{\beta}
$$

Here, we consider the space constructed from $\boldsymbol{a}_{\alpha}$ and $\boldsymbol{a}_{\beta}$, and define the metric tensor:

$$
g_{\alpha \beta}=\boldsymbol{a}_{\alpha} \cdot \boldsymbol{a}_{\beta}
$$

The contravariant vector reciprocal to $a_{\alpha}$ is given by

$$
\boldsymbol{a}^{\alpha}=\sum_{\beta} g^{\alpha \beta} \boldsymbol{a}_{\beta}, \quad g^{\alpha \beta}=\frac{|g|_{\alpha \beta}}{g}, \quad g=\left|g_{\alpha \beta}\right|
$$

where $\left|g_{\alpha \beta}\right|$ and $|g|_{\alpha \beta}$ are the determinant and the cofactor of $g_{\alpha \beta}$, respectively.

The component of "viscosity tensor" is defined from eq 32 :

$$
\eta_{\alpha \beta}=-\left(\frac{1}{\dot{\varepsilon}}\right)^{2} \boldsymbol{v}^{0} \cdot \boldsymbol{F}
$$

This is rewritten with eq 33,35 , and 37 in the form:

$$
\eta_{\alpha \beta}=-\frac{1}{\dot{\varepsilon}} \frac{\boldsymbol{v}^{0}}{\dot{\varepsilon}} \boldsymbol{F}=-\frac{1}{\dot{\varepsilon}} \boldsymbol{a}_{\alpha} \mathscr{F}^{\prime} \boldsymbol{a}_{\beta}
$$

The substitution of eq 39 into eq 43 gives

$$
\eta_{\alpha \beta}=\frac{1}{2} \zeta a_{\alpha} \cdot a_{\beta}-\zeta \sum_{\nu} T_{\alpha}{ }^{\nu} \eta_{\nu \beta}
$$

With the relation

$$
\sum_{\alpha}\left(\eta^{-1}\right)^{\gamma \alpha} \eta_{\alpha \beta}=\delta_{\beta}^{\gamma}
$$

where $\delta_{\beta}^{\gamma}$ is the Kronecker delta, we obtain from eq 44 by the similar calculation used in the previous sections,

$$
\left(\eta^{-1}\right)^{\alpha \beta}=2\left(\frac{g^{\alpha \beta}}{\zeta}+T^{\alpha \beta}\right)
$$

Here we utilize the identity $\eta_{\alpha \beta}=\eta_{\beta \alpha}$, which is derived from that $\boldsymbol{a}_{\alpha} \boldsymbol{T} \boldsymbol{a}_{\beta}=\boldsymbol{a}_{\beta} \boldsymbol{T} \boldsymbol{a}_{\alpha}$.

In the above derivation, we apply the second 
approximation* $^{*}$ to the average of $\left(\boldsymbol{v}^{0} \cdot \boldsymbol{F}\right)$ or $\eta_{\alpha \beta}$; we replace the quantities directly related to the basic vectors by the averaged values of the quantities. Then we have, using eq 45 and 46 ,

$$
\begin{aligned}
\overline{\eta_{\alpha \beta}} & =\frac{-\overline{\left(\eta^{-1}\right)^{\alpha \beta}}}{\left(\overline{\left.\eta^{-1}\right)^{\alpha \alpha}} \overline{\left(\eta^{-1}\right)^{\beta \beta}}-\overline{\left(\eta^{-1}\right)^{\alpha \beta}}\left(\overline{\left.\eta^{-1}\right)^{\beta \alpha}}\right.\right.} \\
& =\frac{1}{\left[4\left(\overline{\frac{g^{\beta \beta}}{\zeta}}+\overline{T^{\beta \beta}}\right)+2\left(\overline{\frac{g^{\alpha \beta}}{\zeta}}+\overline{T^{\alpha \beta}}\right)\right]}
\end{aligned}
$$

Therefore we obtain**

$$
\begin{aligned}
{[\eta]=} & -\frac{N_{0}}{M \eta_{0} \dot{\varepsilon}^{2}} \overline{\overline{\left.\boldsymbol{v}^{0} \cdot \boldsymbol{F}\right)}}=\frac{N_{0}}{M \eta_{0}} \overline{\eta_{\alpha \beta}} \\
= & \frac{N_{0}}{2 M_{\eta_{0}}}\left\{\frac{1}{\zeta \sum_{l=1}^{n}\left\langle\left(\boldsymbol{R}_{l} \boldsymbol{e}_{x}\right)^{2}\right\rangle}+\frac{1}{\left(\sum_{l=1}^{n}\left\langle\left(\boldsymbol{R}_{l} \boldsymbol{e}_{x}\right)^{2}\right\rangle\right)^{2}}\right. \\
& \times \sum_{l=1}^{n} \sum_{s=1}^{n}{ }_{s=1}^{\prime}\left[\left\langle\left(\boldsymbol{R}_{l} \boldsymbol{e}_{x}\right)\left(\boldsymbol{R}_{s} \boldsymbol{e}_{x}\right)\left(\boldsymbol{e}_{y} \boldsymbol{T}_{l s} \boldsymbol{e}_{y}\right)\right\rangle\right. \\
& \left.\left.+\left\langle\left(\boldsymbol{R}_{l} \boldsymbol{e}_{x}\right)\left(\boldsymbol{R}_{s} \boldsymbol{e}_{y}\right)\left(\boldsymbol{e}_{x} \boldsymbol{T}_{l s} \boldsymbol{e}_{y}\right)\right\rangle\right]\right\}^{-1}
\end{aligned}
$$

Taking the average first over all orientations of the molecule, we have

$$
\begin{aligned}
{[\eta]=} & \frac{N_{0}}{6 M \eta_{0}}\left(\zeta \sum_{l=1}^{n}\left\langle R_{l}{ }^{2}\right\rangle\right)\left[1+\frac{\zeta}{8 \pi \eta_{0} \sum_{l=1}^{n}\left\langle R_{l}{ }^{2}\right\rangle}\right. \\
& \times \sum_{l=1}^{n} \sum_{s=1}^{n}\left\{\left\langle\frac{R_{l} R_{s} \cos \alpha_{l s}}{R_{l s}}\right\rangle\right. \\
& +\left\langle\frac { 1 } { 1 0 R _ { l s } ^ { 3 } } \left[ 4 R_{l} R_{s}\left({R_{l}}^{2}+R_{s}{ }^{2}\right) \cos \alpha_{l s}\right.\right. \\
& \left.\left.\left.\left.-R_{l}{ }^{2} R_{s}{ }^{2}\left(1+7 \cos ^{2} \alpha_{l s}\right)\right]\right\rangle\right\}\right]^{-1}
\end{aligned}
$$

where the angular brackets mean an average over internal coordinates only. Application of the second approximation will be proved to be appropriate, in the latter section, by the fact that eq 49 gives the Einstein relation for a sphere on the use of the shell model.

For a rigid molecule composed of $n$ different frictional elements, we obtain as in the previous

* See footnote * on p 619.

** Putting $\boldsymbol{a}_{\alpha}=\sum_{l}\left(\boldsymbol{R}_{l} \boldsymbol{e}_{y}\right) \boldsymbol{e}_{x}$ and $\boldsymbol{a}_{\beta}{ }^{\prime}=\sum_{l}\left(\boldsymbol{R}_{l} \boldsymbol{e}_{x}\right) \boldsymbol{e}_{y}$, we obtain $\overline{\eta_{\alpha \beta}}=\overline{\eta_{\alpha \alpha}^{\prime}}+\overline{\eta_{\alpha \beta}^{\prime}}=1 /\left[\left(\eta^{\prime-1}\right)^{\alpha \alpha}+\left(\eta^{\prime-1}\right)^{\alpha \beta}\right]$, with $\eta_{\alpha \beta}^{\prime}=-\frac{1}{\dot{\varepsilon}} a_{\alpha} \mathscr{F}^{\prime} \boldsymbol{a}_{\beta}^{\prime}$ and $\left(\eta^{\prime-1}\right)^{\alpha \beta}=2\left(\frac{g^{\prime \alpha \beta}}{\zeta}+\right.$ $\left.T^{\prime \alpha \beta}\right)$. Then we have eq 48 also. sections,

$$
\begin{aligned}
& {[\eta]=\frac{N_{0}}{6 M \eta_{0}}\left(\sum_{l=1}^{n} \zeta_{l}\left\langle\boldsymbol{R}_{l}{ }^{2}\right\rangle\right)\left[1+\frac{1}{8 \pi \eta_{0} \sum_{l=1}^{n} \zeta_{l}\left\langle\boldsymbol{R}_{l}{ }^{2}\right\rangle}\right.} \\
& \times \sum_{l=1}^{n} \sum_{s=1}^{n} \zeta_{l} \zeta_{s}\left\{\left\langle\frac{R_{l} R_{s} \cos \alpha_{l s}}{R_{l s}}\right\rangle\right. \\
& +\left\langle\frac { 1 } { 1 0 R _ { l s } ^ { 3 } } \left[ 4 R_{l} R_{s}\left(R_{l}{ }^{2}+R_{s}{ }^{2}\right) \cos \alpha_{l s}\right.\right. \\
& \left.\left.\left.\left.-R_{l}^{2} R_{s}^{2}\left(1+7 \cos ^{2} \alpha_{l s}\right)\right]\right\rangle\right\}\right]^{-1}
\end{aligned}
$$

Here we neglect the effect of Brownian motion. When the molecular form is far from spherical, for example, rod-like, the effect cannot be ignored. In the case of rod-like macromolecules, Riseman and Kirkwood ${ }^{16}$ have assumed that the molecule can be considered as fixed at some definite orientation on account of the overwhelming Brownian motion. The calculation in this case is carried out by replacing $\dot{\varepsilon} / 2$ by $\dot{\varepsilon}$ in the above derivation. This treatment is thought to be inadequate but is used for comparison with the Riseman and Kirkwood result in the latter part. Full consideration of Brownian motion should be given by solving the generalized diffusion equation with respect to the distribution function, as Kirkwood and others ${ }^{10,17}$ have done. However, the difference between the present and the rigorous method appears to be small even in the case of rod-like molecules, as shown later in this work. The rigorous method consists of the derivation of the frictional coefficients by a tensor method and of the intrinsic viscosity from the distribution function. The present unified method, where we use only a tensor method, is obtained by simplification of the neglect of Brownian motion.

\section{Application to "Shell Model"}

Some Preliminary Equations. The "shell model", so called by Bloomfield, ${ }^{1}$ is the method which replaces a particle of given shape by an assembly of small equal frictional elements for example, small equal spheres covering a surface of that shape, without overlap. With the Kirkwood theory and its extension, the translational frictional coefficient of a macromolecule of quite arbitrary shape ${ }^{1}$ and the rotational frictional coefficient of that of cylindrical 
Hydrodynamic Properties of Rigid Complex Molecules

symmetry ${ }^{4}$ have been calculated as the limit of continuous surface shells by increasing the number of small elements.

We consider a macromolecule of molecular weight $M$, composed of $n$ different spherical subunits; each surface shell of a subunit sphere of radius $r_{l}$ consists of $m_{l}$ small frictional elements.

Eq 29 and 49, with removal of angular brackets, become

$$
\begin{aligned}
f_{r}= & \frac{16 \pi \eta_{0}}{3}\left(\sum_{l^{\prime}=1}^{N} R_{l^{\prime}}^{2}\right)^{2} / \sum_{l^{\prime}=1}^{N} \sum_{s^{\prime}=1}^{N} \\
& \times\left(\frac{R_{l^{\prime}}, R_{s^{\prime}} \cos \alpha_{l^{\prime} s^{\prime}}}{R_{l^{\prime} s^{\prime}}}+\frac{R_{l^{\prime}}^{2} R_{s^{\prime}}^{2} \sin ^{2} \alpha_{l^{\prime} s^{\prime}}}{2 R_{l^{\prime} s^{\prime}}^{3}}\right)
\end{aligned}
$$

and

$$
\begin{aligned}
{[\eta]=} & \frac{4 N_{0} \pi}{3 M}\left(\sum_{l^{\prime}=1}^{N} R_{l^{\prime}}^{2}\right)^{2} / \sum_{l^{\prime}=1}^{N} \sum_{s^{\prime}=1}^{N}\left\{\frac{R_{l^{\prime}} R_{s^{\prime}} \cos \alpha_{l^{\prime} s^{\prime}}}{R_{l^{\prime} s^{\prime}}}\right. \\
& +\frac{1}{10 R_{l^{\prime} s^{\prime}}^{3}}\left[4 R_{l^{\prime}} R_{s^{\prime}}\left(R_{l^{\prime}}^{2}+R_{s^{\prime}}^{2}\right) \cos \alpha_{l^{\prime} s^{\prime}}\right. \\
& \left.\left.-R_{l^{\prime}}^{2} R_{s^{\prime}}^{2}\left(1+7 \cos ^{2} \alpha_{l^{\prime} s^{\prime}}\right)\right]\right\}
\end{aligned}
$$

Here, $N$, the total number of the small frictional elements, is large and $\zeta$ vanishes on both the numerator and denominator; $R_{l^{\prime}}, R_{s^{\prime}}, R_{l^{\prime} s^{\prime}}$ $\alpha_{l^{\prime} s^{\prime}}$ are the quantities relating to the small frictional elements and $\sigma$ is the number of the small frictional elements per unit area of the surface shell:

$$
N=\sum_{l=1}^{n} m_{l}, \quad m_{l}=4 \pi \sigma r_{l}^{2}
$$

Case of Single Sphere. For large $N$, the sum in eq 51 and 52 may be replaced by the integral. Using the polar coordinate whose origin locates at the centre of the sphere of radius $r_{0}$, we have

$$
f_{r}=8 \pi \eta_{0} r_{0}{ }^{3}
$$

for the rotational frictional coefficient, which is the Stokes-Kirchhoff law, and

$$
[\eta]=\frac{5 N_{0}}{2 M}\left(\frac{4 \pi r_{0}{ }^{3}}{3}\right)
$$

for the intrinsic viscosity, which is the Einstein relation. ${ }^{7}$

Originally Bloomfield derived Stokes law for the same model from the Kirkwood equation for the translational frictional coefficient. ${ }^{1}$ The relations obtained here correspond with his result.

In the present work, these hydrodynamic relations are used as basic criteria for the characteristics of the fundamental equations.

Effect of Kirkwood-Riseman's approximation. The present calculation employs the Oseen tensor, strictly speaking, the Oseen-BurgersKirkwood tensor, without an approximation such as Kirkwood-Riseman's approximation. ${ }^{14}$ If the similar approximation

$$
\begin{aligned}
& \left\langle\left(\boldsymbol{R}_{l} \boldsymbol{e}_{x}\right)\left(\boldsymbol{R}_{s} \boldsymbol{e}_{x}\right)\left(\boldsymbol{e}_{y} \boldsymbol{T}_{l s} \boldsymbol{e}_{y}\right)\right\rangle+\left\langle\left(\boldsymbol{R}_{l} \boldsymbol{e}_{x}\right)\left(\boldsymbol{R}_{s} \boldsymbol{e}_{y}\right)\left(\boldsymbol{e}_{x} \boldsymbol{T}_{l s} \boldsymbol{e}_{y}\right)\right\rangle \\
& \quad=\left\langle\left(\boldsymbol{R}_{l} \boldsymbol{e}_{x}\right)\left(\boldsymbol{R}_{s} \boldsymbol{e}_{x}\right)\left(\boldsymbol{e}_{y}\left\langle\boldsymbol{T}_{l s}\right\rangle \boldsymbol{e}_{y}\right)\right\rangle \\
& \quad=\left\langle\left(\boldsymbol{R}_{l} \boldsymbol{e}_{x}\right)\left(\boldsymbol{R}_{s} \boldsymbol{e}_{x}\right)\left\langle\boldsymbol{T}_{l s}\right\rangle\right\rangle
\end{aligned}
$$

with

$$
\left\langle\boldsymbol{T}_{l s}\right\rangle=\frac{1}{6 \pi \eta_{0}}\left\langle\frac{1}{R_{l s}}\right\rangle
$$

is adopted, we obtain from eq 28 and 48

$$
f_{r}=\frac{N_{0}}{4 M \eta_{0}[\eta]}
$$

which is the same relation as that proposed previously by Riseman and Kirkwood. ${ }^{15}$

This approximation affects the shell model calculation. Table I shows the result for a sphere of radius $r_{0}$ and a rod of length $L$. The values cited are calculated by the present method except the last two lines, where "Original" means the Oseen-Burgers-Kirkwood tensor" ${ }^{3,14}$ and " $\mathrm{K}-\mathrm{R}$ " means the KirkwoodRiseman approximation.

The two kinds of calculation, "Original" and " $\mathrm{K}-\mathrm{R}$ ", yield the same result for the translational frictional coefficient, because the "Original" tensor is averaged over all orientations of the molecule and becomes equal to the Kirkwood-Riseman approximation. On the other hand, the calculations give different values for the rotational frictional coefficient and the intrinsic viscosity. In particular, in the case of the rotational frictional coefficient of a sphere, the difference amounts to fifty percent of the value obtained from the "Original".

In the rotational frictional coefficient, if we multiply $f_{r}$ of $2 L^{3} \pi \eta_{0} /(9 \cdot \log n)$ by a factor of $3 / 2$ due to the averaging procedure over all 


\section{K. TsudA}

Table I. Comparison of methods of calculation ${ }^{a}$

\begin{tabular}{|c|c|c|c|c|c|}
\hline Model & Method of calculation & $f_{t}$ & $f_{r}$ & {$[\eta]$} & \\
\hline \multirow{2}{*}{ Sphere } & $\begin{array}{l}\text { Tensor } \\
\text { Original }\end{array}$ & $6 \pi \eta_{0} r_{0}$ & $8 \pi \eta_{0} r_{0}{ }^{3}$ & $\frac{5}{2}\left(\frac{4 \pi r_{0}^{3}}{3}\right)$ & \\
\hline & $\mathrm{K}-\mathrm{R} \mathrm{I}$ & $6 \pi \eta_{0} r_{0}$ & $12 \pi \eta_{0} r_{0}^{3}$ & $3 \pi r_{0}^{3} \cdot \frac{N_{0}}{M}$ & \\
\hline \multirow{5}{*}{ Rod } & $\begin{array}{l}\text { Tensor } \\
\text { Original }\end{array}$ & $\frac{n \zeta}{2 \lambda}[\log (n)-(1-2 / \lambda)]^{-1}$ & $\frac{2 L^{3} \pi 0 \eta}{9 \cdot \log (n)}$ & $\frac{10 L^{3} \pi}{252 \cdot \log (n)}$ & $\frac{N_{0}}{M}$ \\
\hline & $\mathrm{K}-\mathrm{R} \mathrm{I}$ & & & $\frac{L^{3} \pi}{24 \cdot \log (n)}$ & $\frac{N_{0}}{M}$ \\
\hline & $\mathrm{K}-\mathrm{R}$ II & $\frac{n \zeta}{2 \lambda}[\log (n)-(1-2 / \lambda)]^{-1}$ & $\frac{L^{3} \pi \eta_{0}}{6 \cdot \log (n)}$ & $\frac{L^{3} \pi}{12 \cdot \log (n)}$ & $\frac{N_{0}}{M}$ \\
\hline & $\begin{array}{l}\text { Rigorous } \\
\text { (Kirkwood and } \\
\text { Auer, }{ }^{10} \text { Sait }^{17} \text { ) }\end{array}$ & & & $\frac{10 L^{3} \pi}{225 \cdot \log (n)}$ & $\cdot \frac{N_{0} \mathrm{~b}}{M}$ \\
\hline & $\begin{array}{c}\text { Riseman and } \\
\left.\text { Kirkwood }{ }^{16}\right) \\
\text { K-R II }\end{array}$ & $\frac{n \zeta}{2 \lambda}[\log (n)-(1-2 / \lambda)]^{-1}$ & $\frac{L^{3} \pi \eta_{0} \mathrm{~b}}{6 \cdot \log (n)}$ & $\frac{L^{3} \pi}{12 \cdot \log (n)}$ & $\frac{N_{0} \mathrm{~b}}{M}$ \\
\hline
\end{tabular}

orientations of the molecule, it reduces to $D^{\theta \theta}$ of $3 k T \cdot \log n / L^{3} \pi \eta_{0}$ which Kirkwood has calcalulated by a tensor method using the rigorous form of the Oseen tensor. ${ }^{2}$

For the viscosity of rod-like molecules, " $\mathrm{K}-$ R I" stands for the values obtained by adopting the rate of $\dot{\varepsilon} / 2$ of rotation of the molecule; this rate is used in all the parts of this report except in the case of " $\mathrm{K}-\mathrm{R}$ II". The " $\mathrm{K}-$ $R$ II" means the results in the case of the overwhelming Brownian motion, ${ }^{16}$ as mentioned in the previous section, which are obtained by replacing $\dot{\varepsilon} / 2$ by $\dot{\varepsilon}$ in calculation of the " $\mathrm{K}-\mathrm{R}$ I'. The values calculated by "Tensor $\mathrm{K}-\mathrm{R}$ II", in the fifth line, are consistent with those obtained by Riseman and Kirkwood in the last line. ${ }^{16}$ Here both methods utilize the KirkwoodRiseman approximation. "Rigorous" denotes the calculation where the Oseen tensor is rigorously taken into account and the effect of Brownian motion is considered in the form of a diffusion equation of distribution function as done by Kirkwood and others. ${ }^{10,17}$ The difference between the value for the "Original" and that for the "Rigorous" by Saito ${ }^{17}$ and Kirkwood and Auer, ${ }^{10}$ may be attributed to Brownian motion. In the last two lines, the values are asymptotic as are Kirkwood and Auer's.

Molecules Composed of Different Spherical Subunits

Rotational Frictional Coefficient. Using the shell model procedure, we calculate a complex macromolecule composed of $n$ spherical subunits, each of which has different size and density. Here, $r_{l}, r_{s}$ are the radius of the $l$ th and $s$ th spherical subunits, whose centre positions locate at $\boldsymbol{R}_{l}$ and $\boldsymbol{R}_{s}$ from the centre of mass of molecule, respectively and $R_{l s}$ is the distance between the centres of the $l$ th and $s$ th subunits.

After lengthy calculation, we obtain from eq 51

$$
f_{r}=\frac{16 \pi \eta_{0} E^{2}}{3(F+G)}
$$

with the abbreviation

$$
\begin{aligned}
& E=4 \sum_{l=1}^{n} r_{l}{ }^{2}\left(r_{l}{ }^{2}+R_{l}{ }^{2}\right) \\
& F=\frac{32}{3} \sum_{l=1}^{n} r_{l}{ }^{3}\left(r_{l}{ }^{2}+2 R_{l}{ }^{2}\right)
\end{aligned}
$$

and

$$
G=\sum_{l=1}^{n} \sum_{s=1}^{n}\left(r_{l} r_{s}\right)^{2}\left(\frac { 1 } { 3 R } \left[36\left(R_{l}^{2}+R_{s}^{2}\right)\right.\right.
$$


Hydrodynamic Properties of Rigid Complex Molecules

$$
\begin{aligned}
& \left.+26\left(r_{l}^{2}+{r_{s}}^{2}\right)-30 R^{2}\right]+\frac{8 R_{l} r_{l}^{2}}{R^{3}} \\
& \times\left(R_{s} \cos \alpha-R_{l}\right)-\frac{8 R_{s} r_{s}{ }^{2}}{R^{3}}\left(R_{s}-R_{l} \cos \alpha\right) \\
& -\frac{\log X}{2 R r_{l} r_{s}}\left[\left(R_{l}{ }^{2}+r_{l}{ }^{2}-R_{s}{ }^{2}-r_{s}{ }^{2}\right)\right. \\
& \left.+\frac{4}{3}\left(R_{l}{ }^{2} r_{l}{ }^{2}+R_{s}{ }^{2} r_{s}{ }^{2}\right)\right]-\frac{R_{l}{ }^{2}}{3 R^{5}}\left[3 \left(R_{s} \cos \alpha\right.\right. \\
& \left.\left.-R_{l}\right)^{2}-R^{2}\right]\left\{-3 R^{2}-5{r_{l}}^{2}+3{r_{s}}^{2}\right. \\
& +\frac{\log X}{4 r_{l} r_{s}}\left[-r_{l}^{2}\left(6 r_{s}^{2}-3 r_{l}^{2}-2 R^{2}\right)\right. \\
& \left.\left.+3\left(R^{2}-r_{s}{ }^{2}\right)^{2}\right]\right\}-\frac{R_{s}{ }^{2}}{3 R^{5}}\left[3\left(R_{s}-R_{l} \cos \alpha\right)^{2}\right. \\
& \left.-R^{2}\right\}\left\{-3 R^{2}-5{r_{s}}^{2}+3 r_{l}{ }^{2}+\frac{\log X}{4 r_{l} r_{s}}\right. \\
& \left.\times\left[-r_{s}{ }^{2}\left(6 r_{l}{ }^{2}-3 r_{s}{ }^{2}-2 R^{2}\right)+3\left(R^{2}-r_{l}{ }^{2}\right)^{2}\right]\right\} \\
& +\frac{4 R_{l}}{R^{3}}\left(R_{l}^{2}+r_{l}^{2}-R_{s}^{2}-r_{s}^{2}\right)\left(R_{s} \cos \alpha\right. \\
& \left.-R_{l}\right)\left[1+\frac{\log X}{4 r_{l} r_{s}}\left(r_{s}^{2}-r_{l}^{2}-R^{2}\right)\right] \\
& +\frac{4 R_{s}}{R^{3}}\left(R_{l}{ }^{2}+{r_{l}}^{2}-R_{s}{ }^{2}-r_{s}{ }^{2}\right)\left(R_{s}-R_{l} \cos \alpha\right) \\
& \times\left[1+\frac{\log X}{4 r_{l} r_{s}}\left(r_{l}{ }^{2}-r_{s}{ }^{2}-R^{2}\right)\right]+\frac{4 R_{l} R_{s}}{R^{5}} \\
& \times\left(R_{s} \cos \alpha-R_{l}\right)\left(R_{s}-R_{l} \cos \alpha\right) \\
& \times\left\{R^{2}+r_{l}^{2}+r_{s}{ }^{2}+\frac{\log X}{4 r_{l} r_{s}}\left[\left(r_{l}{ }^{2}-r_{s}{ }^{2}\right)^{2}\right.\right. \\
& \left.\left.-R^{4}\right]\right\}+\frac{2 R_{l}{ }^{2} R_{s}{ }^{2} \sin ^{2} \alpha}{R^{5}}\left\{R^{2}-r_{l}{ }^{2}-r_{s}{ }^{2}\right. \\
& \left.\left.+\frac{\log X}{4 r_{l} r_{s}}\left[\left(r_{l}+r_{s}\right)^{2}-R^{2}\right]\left[R^{2}-\left(r_{l}-r_{s}\right)^{2}\right]\right\}\right)
\end{aligned}
$$

with

$$
\begin{gathered}
R=R_{l s}, \quad \alpha=\alpha_{l s}, \\
\log X=\log \frac{R^{2}-\left(r_{l}-r_{s}\right)^{2}}{R^{2}-\left(r_{l}+r_{s}\right)^{2}}
\end{gathered}
$$

Here, $F$ and $G$ respectively denote the contribution of the intra-subunit interaction in the $l$ th subunit and the inter-subunit interaction between the $l$ th and $s$ th subunits to the double sum in eq 51 .

Intrinsic Viscosity. The calculation similar to that in the above section leads to

$$
[\eta]=\frac{4 N_{0} \pi E^{2}}{3 M(H+I)}
$$

with the abbreviation

$$
H=\frac{32}{15} \sum_{l=1}^{n} r_{l}{ }^{3}\left(3 r_{l}{ }^{2}+10 R_{l}{ }^{2}\right)
$$

and

$$
\begin{aligned}
& I=\sum_{l=1}^{n} \sum_{s=1}^{n}\left(r_{l} r_{s}\right)^{2}\left(\frac { 1 } { 5 R } \left[52\left(R_{l}{ }^{2}+R_{s}{ }^{2}\right)\right.\right. \\
& \left.+34\left(r_{l}{ }^{2}+r_{s}{ }^{2}\right)-54 R^{2}\right]+\frac{104 R_{l} r_{l}{ }^{2}}{15 R^{3}} \\
& \times\left(R_{s} \cos \alpha-R_{l}\right)-\frac{104 R_{s} r_{s}{ }^{2}}{15 R^{3}} \\
& \times\left(R_{s}-R_{l} \cos \alpha\right)+\frac{\log X}{10 R r_{l} r_{s}}\left[\left(R_{l}^{2}+r_{l}^{2}\right.\right. \\
& \left.\left.-R_{s}{ }^{2}-r_{s}{ }^{2}\right)^{2}+\frac{4}{3}\left(R_{l}{ }^{2} r_{l}{ }^{2}+R_{s}{ }^{2} r_{s}^{2}\right)\right] \\
& +\frac{R_{l}^{2}}{15 R^{5}}\left[3\left(R_{s} \cos \alpha-R_{l}\right)^{2}-R^{2}\right] \\
& \times\left\{-3 R^{2}-5 r_{l}^{2}+3 r_{s}^{2}+\frac{\log X}{4 r_{l} r_{s}}\right. \\
& \left.\times\left[-r_{l}{ }^{2}\left(6 r_{s}{ }^{2}-3 r_{l}{ }^{2}-2 R^{2}\right)+3\left(R^{2}-r_{s}{ }^{2}\right)^{2}\right]\right\} \\
& +\frac{R_{s}{ }^{2}}{15 R^{5}}\left[3\left(R_{s}-R_{l} \cos \alpha\right)^{2}-R^{2}\right] \\
& \times\left\{-3 R^{2}-5 r_{s}{ }^{2}+3 r_{l}{ }^{2}+\frac{\log X}{4 r_{l} r_{s}}\right. \\
& \left.\times\left[-r_{s}{ }^{2}\left(6 r_{l}{ }^{2}-3 r_{s}{ }^{2}-2 R^{2}\right)+3\left(R^{2}-r_{l}{ }^{2}\right)^{2}\right]\right\} \\
& -\frac{4 R_{l}}{5 R^{3}}\left({R_{l}}^{2}+{r_{l}}^{2}-R_{s}{ }^{2}-r_{s}{ }^{2}\right)\left(R_{s} \cos \alpha-R_{l}\right) \\
& \times\left[1+\frac{\log X}{4 r_{l} r_{s}}\left(r_{s}^{2}-r_{l}^{2}-R^{2}\right)\right] \\
& -\frac{4 R_{s}}{5 R^{3}}\left(R_{l}{ }^{2}+{r_{l}}^{2}-{R_{s}}^{2}-{r_{s}}^{2}\right)\left(R_{s}-R_{l} \cos \alpha\right) \\
& \times\left[1+\frac{\log X}{4 r_{l} r_{s}}\left(r_{l}{ }^{2}-r_{s}^{2}-R^{2}\right)\right] \\
& -\frac{4 R_{l} R_{s}}{5 R^{5}}\left(R_{s} \cos \alpha-R_{l}\right)\left(R_{s}-R_{l} \cos \alpha\right) \\
& \times\left\{R^{2}+r_{l}{ }^{2}+{r_{s}}^{2}+\frac{\log X}{4 r_{l} r_{s}}\left[\left(r_{l}{ }^{2}-r_{s}{ }^{2}\right)^{2}\right.\right. \\
& \left.\left.-R^{4}\right]\right\}-\frac{2 R_{l}{ }^{2} R_{s}{ }^{2} \sin ^{2} \alpha}{5 R^{5}}\left\{R^{2}-r_{l}{ }^{2}-r_{s}{ }^{2}\right. \\
& \left.\left.+\frac{\log X}{4 r_{l} r_{s}}\left[\left(r_{l}+r_{s}\right)^{2}-R^{2}\right]\left[R^{2}-\left(r_{l}-r_{s}\right)^{2}\right]\right\}\right)
\end{aligned}
$$


Here, $H, I$ are the terms of the intra-subunit and the inter-subunit interactions respectively; $E, R, \alpha, X$ have the same meaning as in the case of the rotational frictional coefficient. It is remarkable that eq 57 and 62 do not contain any quantities relating to the small frictional elements such as $R_{s^{\prime}}, R_{s^{\prime}}, R_{l^{\prime} s^{\prime}}$.

\section{COMPARISON WITH EXPERIMENT}

The present report deals with bovine serum albumin and $\gamma$ globulin, because the three hydrodynamic properties and fine structures of both molecules have been studied by many researchers.

\section{Bovine Serum Albumin}

The aim of this section is to clarify how the three hydrodynamic properties reflect structural differences in a molecule.

A subunit structure of bovine serum albumin has been suggested in several works, though the structure has not yet been determined conclusively. Weber and Young ${ }^{18}$ have reported two kinds of spherical subunits. They found their radii, $\mathrm{R} I, \mathrm{R}$ II, constant between $\mathrm{pH}$ 2 and 7 and observed $19.8 \AA$ for $\mathrm{R}$ I and $26.9 \AA$ for $\mathrm{R}$ II by diffusion measurement, and $18.8 \AA$ for R I and $26.6 \AA$ for R II by fluorescence depolarization measurements. On the basis of this work, Bloomfield ${ }^{12}$ has recently proposed the model of a covalently bonded trimer, with two spheres of radius $19.0 \AA$, separated by one of radius $26.6 \AA$. For this purpose, he has utilized his extended Kirkwood's equation for the translational frictional coefficient, ${ }^{1}$ the low-angle X-ray scattering theory, and Simha's equation for the intrinsic viscosity. ${ }^{19}$

On the use of the Bloomfield model, calculation of the three hydrodynamic properties is made for three models of bovine serum albumin, slightly different in their structures as shown in Figure 1 and Table II. Here, the molecular weight of 66000 is used as in the case of Bloomfield. ${ }^{12}$ Type $\mathbf{I}$ is the same model as Bloomfield's which has been proposed for the molecular shape at $\mathrm{pH}$ 3.7. All the experimental values cited are those at $\mathrm{pH}$ of about 7 .

The molecule appears more compact at $\mathrm{pH}$ 7 than at low $\mathrm{pH}$. It is deduced that the rotational frictional coefficient linked with the ro-

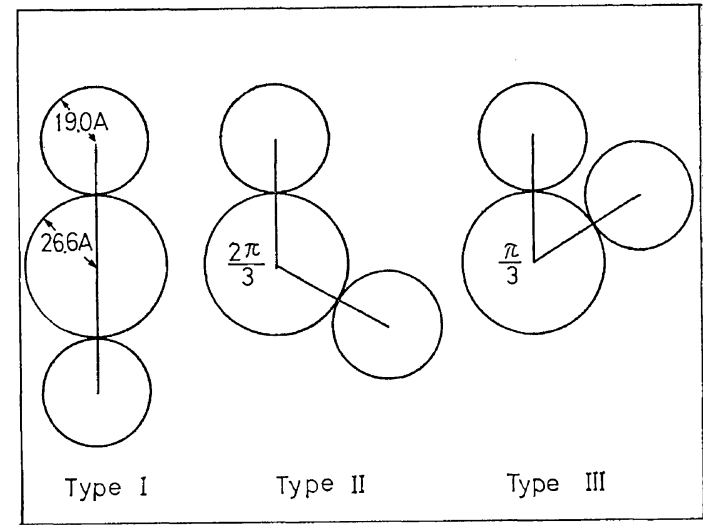

Figure 1. Models for bovine serum albumin.

Table II. Hydrodynamic properties for bovine serum albumin

(at $20^{\circ} \mathrm{C}$ and about neutral $\mathrm{pH}$ )

\begin{tabular}{|c|c|c|c|c|c|}
\hline \multirow[t]{2}{*}{ Calculation } & \multicolumn{2}{|c|}{$\begin{array}{l}D \times 10^{7} \\
\mathrm{~cm}^{2} \mathrm{sec}^{-1}\end{array}$} & \multicolumn{2}{|c|}{$\begin{array}{c}\rho \times 10^{7} \\
\text { sec }\end{array}$} & \multirow{2}{*}{$\frac{\begin{array}{c}{[\eta],} \\
\operatorname{cc~g}^{-1}\end{array}}{\operatorname{Eq~} 62}$} \\
\hline & $\mathrm{Eq} \mathrm{B} 15$ & $\mathrm{Eq} \mathrm{B} 23$ & Eq 31 & Eq 57 & \\
\hline \multicolumn{6}{|l|}{ Model type } \\
\hline$I^{J}$ & 6.11 & 6.06 & 1.45 & 1.91 & 5.46 \\
\hline II & & 6.01 & 1.29 & 1.80 & 5.39 \\
\hline III & & 5.77 & 0.833 & 1.52 & 4.95 \\
\hline \multirow[t]{2}{*}{ Experiment } & \multicolumn{2}{|c|}{$5.84^{20}$} & \multicolumn{2}{|c|}{$1.5^{22}$} & $4.50^{20}$ \\
\hline & \multicolumn{2}{|c|}{$5.93^{21}$} & \multicolumn{2}{|c|}{$1.44^{23}$} & \\
\hline
\end{tabular}

tational relaxation time $\rho$ with the relation $\rho=f_{r} / 2 k T$, is most sensitive to the structural difference of the molecule, among the three hydrodynamic properties; the intrinsic viscosity is less sensitive; the translational frictional coefficient is least.

The values calculated from eq B15 and 31 are listed for comparison with those from equations based on the "shell model", eq 57, 62 and Bloomfield's equation for the translational frictional coefficient:

$$
f_{t}=6 \pi \eta_{0}\left(\sum_{l=1}^{n} r_{l}{ }^{2}\right)^{2}\left(\sum_{l=1}^{n} r_{l}{ }^{3}+\sum_{l=1}^{n} \sum_{s=1}^{n}{ }^{\prime} r_{l}{ }^{2} r_{s}{ }^{2}\left\langle R_{l s}^{-1}\right\rangle\right)^{-1}
$$

The latter are in closer agreement with the experimental values than the former; this difference is remarkable for Type I.

\section{Gamma Globulin}

This section treats a comparison between hydrodynamic models and models determined 
from other procedures such as electron microscopy and X-ray scattering.

We deal with $7 \mathrm{~S} \gamma$ globulin of rabbit, human, pig, and bovine origin. These proteins are heterogeneous as reflected partly in their electrophoretic mobilities, amino acid sequence and composition. This heterogeneity is considered to have close relation to their complicated function of neutralizing others that are harmful. However, all such molecules are suggested to have a common property in that they have the same gross structure and conformation.

Accoding to electron micrographs and lowangle X-ray scattering data, the $\gamma$ globulin molecules are long cylindrical particles. ${ }^{24,25,26}$ They consist of several peptide chains and split in several ways. Reduced in the absence of urea and further treated with urea or acid solution, they dissociate into two types of polypeptide chain referred to as heavy $(\mathrm{H})$ and light $(\mathrm{L})$ chains or as $\mathrm{A}$ and $\mathrm{B}$ chains. ${ }^{27,28,29}$ Mildly reduced and then treated with $\mathrm{HCl}$, they are separated into half molecules. ${ }^{30}$ Hydrolyzed with Papain and reduced, they dissociate into fragments I, II, and III, where the former two and the last are otherwise called fragments $S$ and $F$ respectively. ${ }^{31,32}$ Pepsin digestion yields the fragment closely resembling two $S$ fragments linked by a single disulfide bond.

Many working models of $\gamma$ globulin have been suggested to interpret the course of degradation and the location of the disulfide bonds. Among them, Edelman-Gally's model ${ }^{34}$ is the only model with overall detailed dimensionsan elliptic cylinder with the length of $240 \AA$ and the half axes of the cross section of 9.5 and $28.5 \AA$, which agrees with Kratky and coworkers' low-angle X-ray scattering result for human $\gamma$ globulin. ${ }^{26}$ According to Edelman, the model is based on Kratky's measurements and Almeida and coworkers' electron micrographs for rabbit and goat $\gamma$ globulin. ${ }^{25}$ Therefore, the model may be considered to depict the averaged dimension for human, rabbit, and goat $\gamma$ globulin. Human and rabbit $\gamma$ globulin possess similar but different detailed amino acid compositions; molecular weights of $\mathrm{L}$ chain and $\mathbf{H}$ chain of human $\gamma$ globulin are 20000 and $60000^{29}$ respectively, while those of rabbit are 25000 and 50000 respectively. ${ }^{35}$ These data, as well as other physicochemical data for $\gamma$ globulin, are disagreed among researchers, which reflects the complexity of biological systems.

The present model, closely resembling the Edelman-Gally model, is a rectangular assembly of 36 spheres of radius of $9.5 \AA$, arrayed lengthwise at a separation of $20 \AA$ and breadthwise in contact with each other, whose length, width, and thickness are 239,51 , and $19 \AA$ respectively. This proposed model does not mean that the molecule consists of 36 subunits, but only visualizes the molecule as an assembly of spheres to facilitate the calculation. It differs from the Edelman model in the point that the papain and the pepsin cleavage are depicted in a similar way to the reduction degradations. The model is shown in Figure 2, as well as the course of degradation, which corresponds to Edelman's schematic diagram illustrating the degradation of the $\gamma$ globulin molecule. ${ }^{34}$

Table III shows the calculated values of the rotational relaxation time, the intrinsic viscosity, and the translational frictional coefficient with the molecular weight used; Table IV shows the experimental values-the rotational relaxation time, the intrinsic viscosity, and the translational frictional coefficient calculated by

$$
f_{t}=\frac{(1-\bar{v} d) M}{S N_{0}}
$$

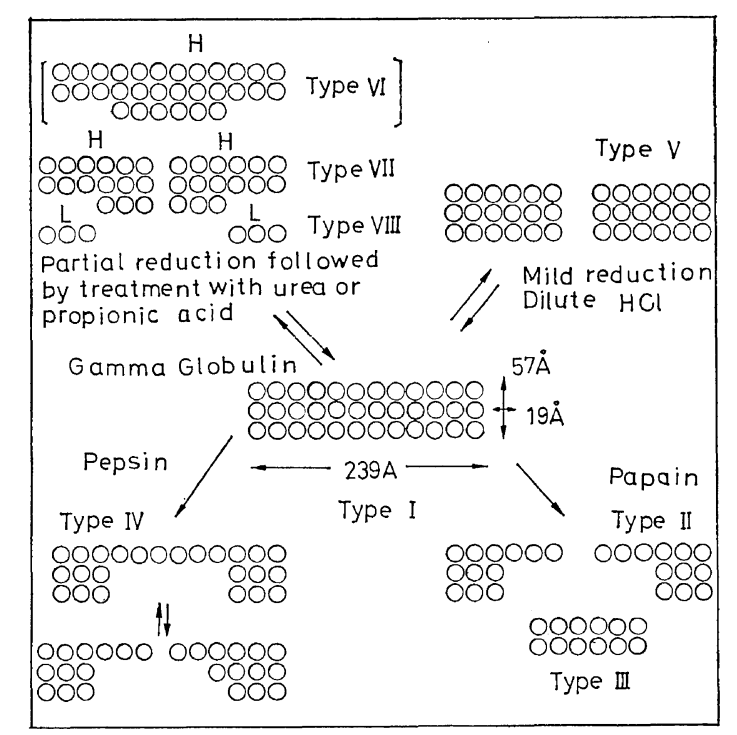

Figure 2. Models for gamma globulin and its split fragments. 
where $S$ is the sedimentation constant, $\bar{v}$ is the partial specific volume of the solute, and $d$ is the density of the solvent. All the experimental values chosen are those at $\mathrm{pH}$ of about 7 .

In papain cleavage of rabbit $\gamma$ globulin, the present theory agrees well with the experiment for both the translational frictional coefficient and the intrinsic viscosity, considering the inequality of the density of $\mathrm{H}$ and $\mathrm{L}$ fragments in the model was not taken into account.

The rotational frictional coefficient calculated for Type $I$ is in the order of $10^{-7}$ and is greater than the experimental values in the order of $10^{-8} \cdot{ }^{36}$ Winkler and co-workers ${ }^{37}$ have reported that the value of $\rho$ for undigested fluorescent rabbit $\gamma$ globulin (Type I) is twice the value

Table III. Calculated values of hydrodynamic properties for gamma globulin (at $20^{\circ} \mathrm{C}$ and about neutral $\mathrm{pH}$ )

\begin{tabular}{rcccc}
\hline $\begin{array}{c}\text { Model } \\
\text { type }\end{array}$ & $\begin{array}{c}f_{t} \times 10^{7}, \\
\mathrm{~g} \mathrm{sec}^{-1}\end{array}$ & $M \times 10^{-5}$ & $\begin{array}{c}{[\eta]} \\
\text { cc g }^{-1}\end{array}$ & $\begin{array}{c}\rho \times 10^{7}, \\
\mathrm{sec}\end{array}$ \\
\hline I & 0.925 & 1.45 & 6.84 & 7.67 \\
II & 0.542 & 0.520 & 3.59 & 1.37 \\
III & 0.540 & 0.480 & 3.50 & 1.27 \\
IV & 0.890 & & & \\
V & 0.616 & & & \\
VI & 0.859 & & & \\
VII & 0.582 & & & \\
VIII & 0.301 & & & \\
\hline
\end{tabular}

found for the fragments prepared by papain digestion and chromatography (a mixture of Type II and III). It does not agree with the present result. The measured relaxation time varies with the fixed fluorescent substance, for example, the time of bovine $\gamma$ globulin is $2.98 \times$ $10^{-7} \mathrm{sec}$ for pyrene and $4.6 \times 10^{-8} \mathrm{sec}$ for napthtyl fluorochrome. $^{38}$ Since the globulin contains little or no $\alpha$-helix, though $\beta$-structure is found, ${ }^{39}$ it may be considered that its component chains are affected by micro-Brownian motion and the fluorescence method does not always reflect the actual macro-rotation of the whole molecule.

Agreement is also close in the case of pepsin digestion.

Most of the measured values reported for separation into half fragments were given at low $\mathrm{pH}$ and only values at neutral $\mathrm{pH}$ were given by Palmer and coworkers. The $3.4 \mathrm{~S}$ subunits formed by reduction and acidification, become the component of $3.7 \mathrm{~S}$ and appear to recombine spontaneously into the component of $5.9 \mathrm{~S}$ in close agreement with the value of the untreated globulin $(6.2 \mathrm{~S}) .^{30}$ Even the component of $3.7 \mathrm{~S}$ does not satisfactorily fit the calculated value for the translational frictional coefficient. The ratio of the coefficient for Type I to that for Type $\mathrm{V}$ is 1.2 , while the calculated ratio is 1.5 .

In the case of reduction followed by treatment

Table IV. Experimental values of hydrodynamic properties for gamma globulin (at $20^{\circ} \mathrm{C}$ and about neutral $\mathrm{pH}$ )

\begin{tabular}{|c|c|c|c|c|c|c|c|}
\hline $\begin{array}{l}\text { Model } \\
\text { type }\end{array}$ & & $\begin{array}{c}S_{\omega} \times 10^{13} \\
\quad \sec \end{array}$ & $M \times 10^{-5}$ & $\begin{array}{c}v \\
\operatorname{cc~}^{-1}\end{array}$ & $\begin{array}{c}f_{t} \times 10^{7} \\
\mathrm{~g} \mathrm{sec}^{-1}\end{array}$ & $\begin{array}{c}{[\eta],} \\
\operatorname{cc~g}^{-1}\end{array}$ & $\begin{array}{c}\rho \times 10^{8}, \\
\sec \end{array}$ \\
\hline \multirow[t]{4}{*}{ I } & Rabbit $^{32}$ & $6.7\left(S_{0}\right)$ & 1.45 & 0.735 & 0.955 & 6.0 & $1^{\mathrm{a}}$ \\
\hline & Human ${ }^{29}$ & 6.9 & 1.60 & 0.73 & 1.0 & $\begin{array}{c}4.8-8.3^{40} \\
\text { (pH } 5.8-7.7)\end{array}$ & \\
\hline & Pig $^{29}$ & 6.7 & & & & & \\
\hline & Bovine & $7.0^{41}$ & & $0.72^{41}$ & & & $0.3-9.0^{36}$ \\
\hline II & Rabbit $^{32}$ & $3.8\left(S_{0}\right)$ & 0.520 & 0.735 & 0.604 & 3.8 & $0.5^{a}$ \\
\hline III & Rabbit $^{32}$ & $3.8\left(S_{0}\right)$ & 0.480 & 0.735 & 0.557 & 4.2 & $0.5^{a}$ \\
\hline IV & Rabbit $^{33}$ & 5.1 & 1.06 & 0.74 & 0.897 & & \\
\hline V & Rabbit $^{30}$ & 3.7 & 0.725 & 0.745 & 0.830 & & \\
\hline VI & Human ${ }^{29}$ & 6.6 & 1.2 & 0.73 & 0.82 & & \\
\hline VII & Pig $^{29}$ & 4.3 & 0.60 & 0.73 & 0.63 & & \\
\hline \multirow[t]{3}{*}{ VIII } & Human $^{29}$ & 3.7 & 0.20 & 0.73 & 0.24 & & \\
\hline & & & 0.23 & 0.73 & 0.28 & & \\
\hline & & & 0.25 & 0.73 & 0.30 & & \\
\hline
\end{tabular}

a Relative values. ${ }^{37}$ 
with urea or propionic acid, the hydrodynamic properties of human and pig $\gamma$ globulin are used. $^{29} \quad$ For heavy chains and their linked fragments, the theory sufficiently explains the experimental value, where the molecular weight of the pig's chain is assumed to be equal to that of the human's. For light chains, the calculations are performed on the basis of molecular weights of 20000, 23000, and 25000; the last being for rabbit but used as a reference.

\section{DISCUSSION}

For a model composed of frictional elements in an arbitrary conformation, we have obtained fundamental equations eq 22,29 , and 49 , extended equations eq 23,31 , and 50 , and eq 57 and 62 for the shell model. Here, eq 22, 29, and 49 are for the model of identical elements and eq 23, 31, 50, 57, and 62 are for the model of different elements. Kirkwood has derived the translational diffusion constant for the above model of identical elements; ${ }^{2}$ Bloomfield also has used the above model of different elements, or different spherical subunits. ${ }^{1}$ With respect to the models, eq 22, 29, and 49 correspond to the result of Kirkwood; eq 23, 31, and 50 correspond to Bloomfield's eq B15; eq 57 and 62 correspond to Bloomfield's eq B23. Here, eq 23 is equal to eq B15.

The present theory is identical to that of Kirkwood in the following reasons. In addition to the use of the same model, both are tensor methods in which the Oseen tensor is adopted rigorously and both take, in the first place, the average over all orientations of the molecule. Furthermore, eq 22 reduces to the Kirkwood equation for the translational diffusion constant. Eq 29 is derived also from the Hearst equation for the rotatory diffusion constants. ${ }^{5}$ In the case of a rod-like molecule, the value obtained by the present method is in agreement with that given by the rigorous method of Kirkwood. ${ }^{2}$ Besides, for the three hydrodynamic properties of rod-like molecules, the present method and the Kirkwood and Riseman theory ${ }^{16}$ give the same result in the Kirkwood-Riseman approximation.

On the other hand, this method is different from Kirkwood's theory in the following respects.
We deal only with a rigid molecule; he deals with a rigid and a flexible molecule. , $^{2,9}$ Our coordinate system is not the same as his system; for example, $D^{\mu \mu}$ means $D^{z z}$ in our system but $D^{\theta \theta}$ in his system. ${ }^{2}$ This is because the basic vectors used vary with the system adopted. Our method treats only the case in which we are allowed to ignore the effect of Brownian motion, while his theory considers the effect fully. The use of a rigid model and the neglect of Brownian motion enable us to obtain the present unified method. We use approximations for the averaging procedure but he does not. Here the approximation is similar to the one adopted by Hearst. ${ }^{5}$ Then we can calculate the rotational frictional coefficient and the intrinsic viscosity as well as the translational frictional coefficient. It is impossible to average the two former hydrodynamic properties without approximation, in contrast to the latter.

The approximation seems to be sufficient for us to derive the following relations which are important criteria in the present work; the above fundamental equations yield the StokesKirchhoff law for the rotational frictional coefficient and the Einstein relation for the intrinsic viscosity for a sphere on the use of the shell model. If we regard as a characteristic of the Kirkwood equation, the fact that the equation gives Stokes law for the same model, the relations derived above show that the present fundamental equations correspond to the Kirkwood equation in these criteria also.

Eq 57 and 62 for the shell model correspond to eq B23, as mentioned above, but do not correspond to Bloomfield's equation for the rotational frictional coefficient of the molecule of cylindrical symmetry. The values calculated from eq 29, 31, 49,50,57, and 62 depend on the location of the molecular centre of mass, while the values from eq B15 and B23 do not. For a single sphere, eq 57 and 62 give the Stokes-Kirchhoff law and the Einstein relation respectively, while eq 29 and 49 yield zero, but both of eq B15 and B23 give Stokes law. From the model adopted and the method of derivation, it is probable that eq 29 and 49 have defects similar to those of Kirkwood's equation corresponding to eq 22, which has been previouly pointed out: ${ }^{43}$ eq 57 and 62 contain faults 
similar to those of eq B 23; that is, the value obtained with eq 23 has been shown by Bloomfield to deviate a little from Perrin's result for ellipsoids. ${ }^{1}$

In the case of bovine serum albumin, a compact trimer model is best fitted to the experimental data and the sensitivity of the hydrodynamic properties to structural difference arranged in order of increasing sensitivity is the translational frictional coefficient, the intrinsic viscosity, and the rotational frictional coefficient. The equations for the shell model agree much better with the experiments than the extended equations. In the case of $\gamma$ globulin, the Edelman-Gally model explains consistently the hydrodynamic behaviour of the molecule and its subunits in various stages of degradation, except a few cases.

It is concluded that the equations for the shell model, extended from the fundamental equations, apply sufficiently well to rigid complex molecules except for some discrepancies mentioned above.

Acknowledgements. The author wishes to express his gratitude to Professor M. Kurata of Kyoto University for his interest and encouragement, and to Professor T. Suzuta of Tokyo Medical College for helpful discussions concerning biological molecules.

\section{REFERENCES}

1. V. A. Bloomfield, W. O. Daltan, and K. E. Van Holde, Biopolymers, 5, 135 (1967).

2. J. Riseman and J. G. Kirkwood, "Rheology", Vol. I.E.R. Eirich, Ed. Academic Press, New York, N.Y., 1956, p 495.

3. J. M. Burgers, "Second Report on Viscosity and Plasticity", Amsterdam Acad. Sci., Nordemann, Amsterdam, 1938, Chap. 3.

4. D. P. Filson and V. A. Bloomfield, Biochemistry, 6, 1650 (1967).

5. J. E. Hearst, J. Chem. Phys., 38, 1062 (1963).

6. K. Tsuda, Bull. Chem. Soc. Japan, 42, 850 (1969); Details to be published in Rheol. Acta; K. Tsuda, Abstract, SPSJ 17th Symposium, on Macromolecules, Matsuyama, October, 1968.

7. A. Einstein, Ann. Phys., 19, 289 (1906); ibid., 34, 591 (1911).

8. J. G. Kirkwood, Rec. Trav. Chim., 68, 649 (1946).
9. J. G. Kirkwood, J. Polym. Sci., 12, 1 (1954).

10. J. G. Kirkwood and P. L. Auer, J. Chem. Phys., 19, 281 (1951).

11. V. A. Bloomfield, K. E. Van Holde, and W. O. Daltan, Biopolymers, 5, 149 (1967).

12. V. A. Bloomfield, Biochemistry, 5, 684 (1966).

13. K. Tsuda, Sen-i-koygo Shikenjo Kenkyuhokoku, No. 83, 1 (1968).

14. J. G. Kirkwood and J. Riseman, J. Chem. Phys., 16, 565 (1948).

15. J. Riseman and J. G. Kirkwood, J. Chem. Phvs., 17, 442 (1949).

16. J. Riseman and J. G. Kirkwood, J. Chem. Phys., 18, 512 (1950).

17. N. Saito, J. Phys. Soc. Japan, 6, 302 (1951).

18. G. Weber and L. B. Young, J. Biol. Chem., 239, 1415, 1425 (1964).

19. R. Simha, J. Phys. Chem., 44, 25 (1940).

20. M. Champagne, J. Chim. Phys., 54, 378 (1957).

21. W. F. Harrington, P. J. Johnson, and R. H. Ottewill, Biochem. J., 62, 569 (1959).

22. R. Steiner and A. McAlister, J. Polym. Sci., 24, 105 (1957).

23. G. Weber, Biochem. J., 51, 155 (1952).

24. C. E. Hall, A. Nisonoff, and H. S. Slayter, J. Biophys. Biochem. Cytol., 6407 (1959).

25. J. Almeida, B. Cinader, and A. Howatson, J. Exptl. Med., 118, 327 (1963).

26. O. Kratky, G. Porod, A. Sekora, and B. Palette, J. Polym. Sci., 16, 163 (1955); O. Kratky and B. Paletta, Angew. Chem., 67, 602 (1955); O. Kratky, I. Pilz, P. J. Schmitz, and R. Oberdorfer, Z. Naturforsch., 18b, 180 (1963).

27. G. M. Edelman and M. D. Poulik, J. Exptl. Med., 113, 961 (1961).

28. J. B. Fleishman, R. R. Porter, and E. M. Press, Biochem. J., 88, 220 (1963).

29. D. E. Olins and G. M. Edelman, J. Exptl. Med., 119, 789 (1964).

30. J. L. Palmer, A. Nisonoff, and K. E. Van Holde, Proc. Natl. Acad. Sci. U.S., 50, 314 (1963).

31. R. R. Porter, Biochem. J., 73, 119 (1959).

32. M. E. Noelken, C. A. Nelson, C. E. Buckley, and C. Tanford, J. Biol, Chem., 240, 218 (1965).

33. A. Nisonoff, G. Markus, and F.C. Wissler, Nature, 189, 293 (1961).

34. G. M. Edelman and J. A. Gally, Proc. Natl. Acad. Sci. U.S., 51, 846 (1964).

35. E. Marler, C. A. Nelson, and C. Tanford, Biochemistry, 3, 279 (1964).

36. F. H. Chowdhury and P. Johnson, Biochim. Biophys. Acta, 66, 218 (1963).

37. M. H. Winkler, M. B. Goldman, and E. A. 


\section{Hydrodynamic Properties of Rigid Complex Molecules}

Sweeney, Biochim. Biophys. Acta, 112, 559 41. E. L. Hess and H. F. Deutsch, J. Amer. Chem. (1966). Soc., 70, 84 (1948).

38. C. S. Chadwick and P. Johnson, Biochim. 42. M. O. Dayhoff, G. Perlmann, and D. A. Biochys. Acta, 53, 482, (1961).

39. K. Imahori, Biopolymers, 1, 563 (1963). MacInnes, J. Amer. Chem. Soc., 74, 2515 (1952).

40. B. Jirgensons and E. C. Adams, Makromol. 43. For example, R. Zwanzig, J. Chem. Phys., 45, Chem., 24, 194 (1957). 1858 (1966). 